\title{
A Deduction of the Hellmann-Feynman Theorem
}

\section{Chen Feng ${ }^{1}$. Cheng Wei ${ }^{1} \cdot$ Bao-long Fang ${ }^{1} \cdot$ Hong-yi Fan ${ }^{2}$}

Received: 30 June 2019 / Accepted: 11 December 2019/ Published online: 28 March 2020

(C) Springer Science+Business Media, LLC, part of Springer Nature 2020

\begin{abstract}
In this paper we present a deduction of the Hellmann-Feynman (HF) theorem for the lowest eigenenergy $E_{0}(\lambda)$ of a Hamiltonian $H(\lambda)$, that is : its second-order derivative with respect to he parameter $\lambda, \frac{\partial^{2} E_{0}}{\partial \lambda^{2}}$, is always less than the expectation value of $\frac{\partial^{2} H(\lambda)}{\partial \lambda^{2}}$ in the ground state. We also point out that the above deduction does not hold for the FH theorem in ensemble average. The electric polarizability of molecules is studied by the deduction of the HF theorem
\end{abstract}

Keywords Hellmann-Feynman (HF) theorem · Eigenenergy $E_{0}(\lambda) \cdot$ Hamiltonian $H(\lambda)$. The expectation value

\section{Introduction}

In theoretical quantum physics and quantum chemistry the Hellmann-Feynman (HF) theorem $[1,2]$ has been widely used for calculating various observables [3-6]. The HellmannFeynman theorem states that when a system's Hamiltonian, which depends upon a real parameter $\lambda$, possesses its energy eigenvector $\left|\alpha_{n}\right\rangle, H\left|E_{n}\right\rangle=E_{n}\left|E_{n}\right\rangle$ with $\left\langle E_{n} \mid E_{n}\right\rangle=1$, then

$$
\frac{\partial E_{n}(\lambda)}{\partial \lambda}=\frac{\partial\left\langle E_{n}|H(\lambda)| E_{n}\right\rangle}{\partial \lambda}=\left\langle E_{n}\left|\frac{\partial H}{\partial \lambda}\right| E_{n}\right\rangle,
$$

Applying the HF theorem to multi-electron-neucleon interaction one can derive the electrostatic theorem [5]. By noticing that the HF theorem only deals with the first-order derivative of the average energy (or $H(\lambda)$ ) with respect to $\lambda$, an interesting question thus naturally arises: is there any physical rule or physical meaning which can be exposed by performing

\footnotetext{
Work supported by the Natural Science Fund of Education Department of Anhui province(KJ2016A590), The talent foundation of Hefei University(15RC11), the Natural Science Foundation of the Anhui Higher Education Institutions of China(KJ2016SD49),Hefei university academic leader(2016dtr02)
}

Chen Feng

chenfeng@hfuu.edu.cn

1 Department of Mathematics and Physics, Hefei University, Hefei, Anhui 230022, China

2 Department of Material Science and Engineering, University of Science and Technology of China, Hefei, Anhui 230026, People's Republic of China 
the second-order derivative of $E(\lambda)$ with respect to the parameter $\lambda$ ? This question has some physical background, for instance, the electric polarizability, denoted as $\alpha$ in this paper, of a molecule is a measure of its ability to respond to an electric field and acquires an electric dipole moment, $\vec{\mu}=\sum_{i} q_{i} \vec{r}_{i}$, where $q_{i}$ is the charge of the particle $i$ at the location $\vec{r}_{i}$. The effect caused by an electric field $\vec{\varepsilon}$, applied in the $x$-direction with electric field strength $\varepsilon$, which is assumed uniform over the molecule, is described by

$$
H=H_{0}-\vec{\mu} \cdot \vec{\varepsilon}=H_{0}-\mu_{x} \varepsilon, \mu_{x}=q X,
$$

where $H_{0}$ denotes the Hamiltonian of a molecule in the absence of the field, which is usually taken as

$$
H_{0}=\frac{P^{2}}{2 m}+\frac{m \omega^{2}}{2} X^{2} .
$$

In the context of quamtum mechanics, the expectation value of the electric dipole moment operator $\mu_{x}$ in the presence of the electric field is the sum of a permanent dipole moment $\mu_{0 x}$ and the contribution induced by the field, so we can expand

$$
\left\langle\mu_{x}\right\rangle=\mu_{0 x}+\alpha_{x x} \varepsilon+O\left(\varepsilon^{2}\right)+\cdots,
$$

where $\alpha_{x x}$ is named the polarizability in the $x$-direction [6]. For obtaining the polarizability, one may use the HF theorem such that the variation of the energy $E_{n}$ with respect to the electric field strength $\varepsilon$ is given by

$$
\frac{d E_{n}}{d \varepsilon}=\left\langle\psi_{n}\left|\frac{\partial H}{\partial \varepsilon}\right| \psi_{n}\right\rangle=-\left\langle\psi_{n}\left|\mu_{x}\right| \psi_{n}\right\rangle, H\left|\psi_{n}\right\rangle=E_{n}\left|\psi_{n}\right\rangle .
$$

On the other hand, the energy $E_{n}$ of the molecule in the presence of the electric field can be Taylor-expanded relative to its energy $E_{n}(0)$ in the absence of the field

$$
E_{n}=E_{n}(0)+\left(\frac{d E_{n}}{d \varepsilon}\right)_{0} \varepsilon+\frac{1}{2}\left(\frac{d^{2} E_{n}}{d \varepsilon^{2}}\right)_{0} \varepsilon^{2}+\frac{1}{3 !}\left(\frac{d^{3} E_{n}}{d \varepsilon^{3}}\right)_{0} \varepsilon^{3}+\cdots
$$

the subscript 0 implies that the derivative is evaluated at $\varepsilon=0$. Substituting (6) into (4) yields

$$
-\frac{d E_{n}}{d \varepsilon}=\left\langle\psi_{n}\left|\mu_{x}\right| \psi_{n}\right\rangle=-\left(\frac{d E_{n}}{d \varepsilon}\right)_{0}-\left(\frac{d^{2} E_{n}}{d \varepsilon^{2}}\right)_{0} \varepsilon-\frac{1}{2}\left(\frac{d^{3} E_{n}}{d \varepsilon^{3}}\right)_{0} \varepsilon^{2}-\cdots,
$$

Then comparing (7) with (5) one can identify

$$
\mu_{0 x}=-\left(\frac{d E_{n}}{d \varepsilon}\right)_{0}, \quad \alpha_{x x}=-\left(\frac{d^{2} E_{n}}{d \varepsilon^{2}}\right)_{0}, \quad \ldots
$$

thus for obtaining $\alpha_{x x}$ we need to enlarge the scope of usual FH theorem to the case about the second-order derivative $\frac{d^{2} E_{n}}{d \varepsilon^{2}}$ and explore its relation to $\frac{d^{2} H}{d \varepsilon^{2}}$. In the following we shall analyse it and will obtain a deduction of the usual FH theorem, that is:

For the lowest eigenenergy $E_{0}(\lambda)$, its second-order derivative with respect to parameter $\lambda$ is always less than the expectation value of $\frac{\partial^{2} H(\lambda)}{\partial \lambda^{2}}$ in the ground state.

In Ref. [7] Fan and Chen has developed the HF theorem for pure state to mixed state case, we shall also point out that the above deduction does not hold for the FH theorem in ensemble avarage case. 


\section{Deduction of the FH Theorem}

Writing the eigenvalue equation of $H$ as $\left(H-E_{n}\right)\left|E_{n}\right\rangle \equiv G_{n}\left|E_{n}\right\rangle=0$,

$$
\left(H-E_{n}\right) \equiv G_{n} \text {. }
$$

by considering that this equation holds for any variation of $\lambda$, we have

$$
\frac{\partial G_{n}}{\partial \lambda}\left|E_{n}\right\rangle+G_{n} \frac{\partial}{\partial \lambda}\left|E_{n}\right\rangle=0,
$$

From the hermitian of the Hamiltonian operator $H$, it follows

$$
\left\langle E_{n}\right| \frac{\partial G_{n}}{\partial \lambda}+\frac{\partial\left\langle E_{n}\right|}{\partial \lambda} G_{n}=0 .
$$

Then

$$
\left(\left\langle E_{n}\right| \frac{\partial G_{n}}{\partial \lambda}\right) \frac{\partial}{\partial \lambda}\left|E_{n}\right\rangle=\left(-\frac{\partial\left\langle E_{n}\right|}{\partial \lambda} G_{n}\right) \frac{\partial}{\partial \lambda}\left|E_{n}\right\rangle .
$$

Doing the second-order derivative with respect to $\lambda$ for (10) leads to

$$
\frac{\partial^{2} G_{n}}{\partial \lambda^{2}}\left|E_{n}\right\rangle+2 \frac{\partial G_{n}}{\partial \lambda} \frac{\partial}{\partial \lambda}\left|E_{n}\right\rangle+G_{n} \frac{\partial^{2}}{\partial \lambda^{2}}\left|E_{n}\right\rangle=0,
$$

then taking inner product with $\left\langle E_{n}\right|$ we have

$$
\left\langle E_{n}\left|\frac{\partial^{2} G_{n}}{\partial \lambda^{2}}\right| E_{n}\right\rangle+2\left\langle E_{n}\left|\frac{\partial G_{n}}{\partial \lambda} \frac{\partial}{\partial \lambda}\right| E_{n}\right\rangle+\left\langle E_{n}\left|G_{n} \frac{\partial^{2}}{\partial \lambda^{2}}\right| E_{n}\right\rangle=0 .
$$

After taking $\left\langle E_{n}\right| G_{n}=0$ into account, (14) becomes to

$$
\left\langle E_{n}\left|\frac{\partial^{2} G_{n}}{\partial \lambda^{2}}\right| E_{n}\right\rangle=-2\left\langle E_{n}\left|\frac{\partial G_{n}}{\partial \lambda} \frac{\partial}{\partial \lambda}\right| E_{n}\right\rangle
$$

then from (12) we have

$$
\left\langle E_{n}\left|\frac{\partial^{2} G_{n}}{\partial \lambda^{2}}\right| E_{n}\right\rangle=2\left(\frac{\partial\left\langle E_{n}\right|}{\partial \lambda}\right) G_{n} \frac{\partial}{\partial \lambda}\left|E_{n}\right\rangle
$$

or

$$
\frac{\partial^{2} E_{n}}{\partial \lambda^{2}}=\left\langle E_{n}\left|\frac{\partial^{2} H}{\partial \lambda^{2}}\right| E_{n}\right\rangle-2\left(\frac{\partial\left\langle E_{n}\right|}{\partial \lambda}\right)\left(H-E_{n}\right)\left(\frac{\partial\left|E_{n}\right\rangle}{\partial \lambda}\right),
$$

which is sharply in contrast to (1), i.e., while one has $\frac{\partial E_{n}(\lambda)}{\partial \lambda}=\left\langle E_{n}\left|\frac{\partial H}{\partial \lambda}\right| E_{n}\right\rangle$, one should know $\frac{\partial^{2} E_{n}(\lambda)}{\partial \lambda^{2}} \neq\left\langle E_{n}\left|\frac{\partial^{2} H}{\partial \lambda^{2}}\right| E_{n}\right\rangle$. By setting

we see

$$
|\psi\rangle_{n}=\frac{\partial}{\partial \lambda}\left|E_{n}\right\rangle
$$

$$
\frac{\partial^{2} E_{n}}{\partial \lambda^{2}}=\left\langle E_{n}\left|\frac{\partial^{2} H}{\partial \lambda^{2}}\right| E_{n}\right\rangle-2_{n}\left\langle\psi\left|\left(H-E_{n}\right)\right| \psi\right\rangle_{n} .
$$

In particular, if $E_{n}$ is the ground state energy $E_{0}$, then the value $0\langle\psi|H| \psi\rangle_{0}-E_{0}=$ $\left(\frac{\partial}{\partial \lambda}\left\langle E_{0}\right|\right) H\left(\frac{\partial}{\partial \lambda}\left|E_{0}\right\rangle\right)-E_{0} \geqslant 0$, is always positive, so

$$
\frac{\partial^{2} E_{0}}{\partial \lambda^{2}}=\left\langle E_{0}\left|\frac{\partial^{2} H}{\partial \lambda^{2}}\right| E_{0}\right\rangle-2_{0}\left\langle\psi\left|\left(H-E_{0}\right)\right| \psi\right\rangle_{0} \leqslant\left\langle E_{0}\left|\frac{\partial^{2} H}{\partial \lambda^{2}}\right| E_{0}\right\rangle,
$$

this states that for the lowest energy $E_{0}$, its second-order derivative with respect to $\lambda, \frac{\partial^{2} E_{0}}{\partial \lambda^{2}}$, is always less than the expectation value of $\frac{\partial^{2} H}{\partial \lambda^{2}}$ in the ground state, this is the deduction of the Hellmann-Feynman theorem. 
For example, for a harmonic oscillator $H_{0}=\frac{P^{2}}{2 m}+\frac{m \omega^{2}}{2} X^{2}$, in its ground state $|0\rangle$ with the energy $E_{0}=\hbar \omega$, we do have

$$
\left\langle 0\left|\frac{\partial^{2} H_{0}}{\partial \omega^{2}}\right| 0\right\rangle=\left\langle 0\left|m X^{2}\right| 0\right\rangle=\frac{m}{2}\left\langle 0\left|\left(a+a^{\dagger}\right)^{2}\right| 0\right\rangle \frac{\hbar}{m \omega}=\frac{\hbar}{2 \omega} \geq \frac{\partial^{2} E_{0}}{\partial \omega^{2}}=0,
$$

where $X=\sqrt{\frac{\hbar}{2 m \omega}}\left(a+a^{\dagger}\right)$, and noticing $P=i \sqrt{\frac{m \omega \hbar}{2}}\left(a^{\dagger}-a\right)$ we also have

$$
\left\langle 0\left|\frac{\partial^{2} H_{0}}{\partial m^{2}}\right| 0\right\rangle=\left\langle 0\left|\frac{P^{2}}{m^{3}}\right| 0\right\rangle=\frac{-\omega \hbar}{2 m^{2}}\left\langle 0\left|\left(a-a^{\dagger}\right)^{2}\right| 0\right\rangle=\frac{\omega \hbar}{2 m^{2}} \geq \frac{\partial^{2} E_{0}}{\partial m^{2}}=0,
$$

satisfying (20). We now apply (20) to studying the Electric polarizability of a molecule described by the Hamiltonian in (2), we have

$$
\left\langle\frac{\partial H}{\partial \varepsilon}\right\rangle_{n}=\langle-q X\rangle_{n}=\frac{\partial E_{n}(\varepsilon)}{\partial \varepsilon} .
$$

Supposing that the bound eigenstate of $H=H_{0}-\mu_{x} \varepsilon$ is $|n\rangle$ (not $H_{0}$ 's eigenstate), from $[X, P]=i \hbar$, we have

$$
0=\left\langle n\left|\frac{1}{i \hbar}[P, H]\right| n\right\rangle=\left\langle n\left|\left(q \varepsilon-m \omega^{2} X\right)\right| n\right\rangle .
$$

It follows

$$
\langle n|X| n\rangle=\frac{q \varepsilon}{m \omega^{2}},
$$

and

$$
\frac{\partial E_{n}(\varepsilon)}{\partial \varepsilon}=-\frac{q^{2} \varepsilon}{m \omega^{2}}
$$

which is independent of $n$, so we can write

$$
E(\varepsilon)=-\frac{q^{2} \varepsilon^{2}}{2 m \omega^{2}}
$$

As a result of (8), the polarizability is

$$
\alpha_{x x}=-\left(\frac{d^{2} E}{d \varepsilon^{2}}\right)_{0}=\frac{q^{2}}{m \omega^{2}} .
$$

We now check if (28) agrees with (20), comparing with (20) we see indeed

$$
\frac{d^{2} E}{d \varepsilon^{2}}=\frac{-q^{2}}{m \omega^{2}} \leqslant\left\langle E_{0}\left|\frac{\partial^{2} H}{\partial \varepsilon^{2}}\right| E_{0}\right\rangle=0,
$$

since $\frac{\partial^{2} H}{\partial \varepsilon^{2}}=0$. Further, from (20) we also obtain

$$
\frac{-q^{2}}{m \omega^{2}}=-2_{n}\left\langle\psi\left|\left(H-E_{n}\right)\right| \psi\right\rangle_{n}, \quad|\psi\rangle_{n}=\frac{\partial}{\partial \varepsilon}\left|E_{n}\right\rangle
$$

Since the Hamiltonian in (2) can be rewritten as

$$
H=H_{0}-\mu_{x} \varepsilon=\frac{P^{2}}{2 m}+\frac{m \omega^{2}}{2} X^{2}-\varepsilon q X=\omega a^{\dagger} a-\varepsilon q \sqrt{\frac{\hbar}{2 m \omega}} \frac{a^{\dagger}+a}{\sqrt{2}},
$$

its eigenstate $\left|E_{n}\right\rangle$ is a coherent state. If $|\psi\rangle_{n}$ is normalizable, ${ }_{n}\langle\psi \mid \psi\rangle_{n}=c$, we have the expectation value of $H$ in $|\psi\rangle_{n}$

$$
{ }_{n}\langle\psi|H| \psi\rangle_{n}=\frac{q^{2}}{2 m \omega^{2}}+c E_{n} .
$$




\section{Some Discussions}

We now consider two moleculars with mutual interaction in electric field described by the Hamiltonian

$$
H_{2}=\sum_{i=1}^{2}\left(\frac{P_{i}^{2}}{2 m}+\frac{m \omega^{2}}{2} X_{i}^{2}\right)-\varepsilon q\left(X_{1}+X_{2}\right)-\lambda X_{1} X_{2}
$$

We calculate for the eigenvector of $\mathrm{H}_{2}$

$$
\begin{aligned}
\left\langle\psi_{n}\left|X_{1}\right| \psi_{n}\right\rangle & =\frac{1}{i \hbar}\left\langle\psi_{n}\left|\left[P_{1}, H_{2}\right]\right| \psi_{n}\right\rangle=\left\langle\psi_{n}\left|\left(\varepsilon q-m \omega^{2} X_{1}+\lambda X_{2}\right)\right| \psi_{n}\right\rangle \\
\left\langle\psi_{n}\left|X_{2}\right| \psi_{n}\right\rangle & =\frac{1}{i \hbar}\left\langle\psi_{n}\left|\left[P_{2}, H_{2}\right]\right| \psi_{n}\right\rangle=\left\langle\psi_{n}\left|\left(\varepsilon q-m \omega^{2} X_{2}+\lambda X_{1}\right)\right| \psi_{n}\right\rangle
\end{aligned}
$$

noting

$$
\left\langle\psi_{n}\left|\left[P_{i}, H_{2}\right]\right| \psi_{n}\right\rangle=0, i=1,2
$$

so

$$
\left\langle\psi_{n}\left|\left(X_{1}+X_{2}\right)\right| \psi_{n}\right\rangle=\frac{2 q \varepsilon}{m \omega^{2}-\lambda}
$$

Thus the variation of the energy $E_{n}$ with respect to the electric field strength $\varepsilon$ is

$$
\frac{d E_{n}}{d \varepsilon}=-\left\langle\psi_{n}\left|q\left(X_{1}+X_{2}\right)\right| \psi_{n}\right\rangle=-\frac{2 q^{2} \varepsilon}{m \omega^{2}-\lambda}
$$

which is not related to $n$, and the polarizability is

$$
\alpha_{x x}=-\left(\frac{d^{2} E}{d \varepsilon^{2}}\right)_{0}=\frac{2 q^{2}}{m \omega^{2}-\lambda}
$$

hence we see when two moleculars has inner interaction, its polarizability in electric field becomes to $\frac{2 q^{2}}{m \omega^{2}-\lambda}$. Moreover, when $m \omega^{2}>\lambda$, we do have

$$
\frac{d^{2} E_{0}}{d \varepsilon^{2}}=-\frac{2 q^{2}}{m \omega^{2}-\lambda} \leqslant\left\langle E_{0}\left|\frac{\partial^{2} H_{2}}{\partial \varepsilon^{2}}\right| E_{0}\right\rangle=0,
$$

which conforms to the deduction of Hellmann-Feynman theorem.

By noticing that the usual Hellmann-Feynman theorem is about the pure state average $\left\langle\psi_{n}\left|\frac{\partial H}{\partial \lambda}\right| \psi_{n}\right\rangle$, Fan and Chen developed it to the case of ensemble average (denoted by \langle\rangle$_{e}$ ) and derived the generalized Hellmann-Feynman (GFHT) in Refs. [7-11]

$$
\frac{d}{d \lambda}\langle H\rangle_{e} \equiv \frac{d \bar{E}}{d \lambda}=\left\langle(1+\beta \bar{E}-\beta H) \frac{\partial H}{\partial \lambda}\right\rangle_{e}
$$

where $\langle H\rangle_{e} \equiv \bar{E}$ indicates the ensemble average, $\beta=1 / K T, K$ is the Boltzmann constant. When $H$ is $\beta$-independent, (39) becomes to [7]

$$
\frac{d}{d \lambda}\langle H(\lambda)\rangle_{e}=\left(1+\beta \frac{\partial}{\partial \beta}\right)\left\langle\frac{\partial H}{\partial \lambda}\right\rangle_{e}=\frac{\partial}{\partial \beta}\left[\beta\left\langle\frac{\partial H}{\partial \lambda}\right\rangle_{e}\right] .
$$

However, we do not have a general conclusion that $\frac{\partial^{2} E_{n}}{\partial \lambda^{2}} \leqslant\left\langle E_{n}\left|\frac{\partial^{2} H}{\partial \lambda^{2}}\right| E_{n}\right\rangle$ for all $n$, so the above deduction does not hold for the ensemble avarage.

For example, when

$$
H^{\prime}=\left(\begin{array}{ll}
0 & \lambda \\
\lambda & 1
\end{array}\right),
$$


so

$$
\frac{\partial^{2} H^{\prime}}{\partial \lambda^{2}}=0,\left\langle E_{n}\left|\frac{\partial^{2} H^{\prime}}{\partial \lambda^{2}}\right| E_{n}\right\rangle \equiv 0
$$

On the other hand, $H^{\prime}$ eigenvalues are

$$
E_{0}=\frac{1-\sqrt{1+4 \lambda^{2}}}{2}, E_{1}=\frac{1+\sqrt{1+4 \lambda^{2}}}{2}
$$

it follows

$$
\frac{\partial^{2} E_{0}}{\partial \lambda^{2}}=-\frac{4 \lambda^{2}}{\left(1+4 \lambda^{2}\right)^{3 / 2}}<0,
$$

which obeys the deduction of $\mathrm{FH}$ theorem, however, for $E_{1}$ we can calculate

$$
\frac{\partial^{2} E_{1}}{\partial \lambda^{2}}=\frac{4 \lambda^{2}}{\left(1+4 \lambda^{2}\right)^{3 / 2}}>0
$$

which does not obey the deduction of $\mathrm{FH}$ theorem.

In summary, we have presented the deduction of the Hellmann-Feynman (HF) theorem for the lowest eigenenergy $E_{0}(\lambda)$ of a Hamiltonian $H(\lambda)$, that is : its second-order derivative with respect to he parameter $\lambda, \frac{\partial^{2} E_{0}}{\partial \lambda^{2}}$, is always less than the expectation value of $\frac{\partial^{2} H(\lambda)}{\partial \lambda^{2}}$ in the ground state. The electric polarizability of molecules is studied by the deduction of the HF theorem.

\section{References}

1. Hellmann, H.: Einfuehrung in Die Quantenchemie. Deuticke, Leipzig (1937)

2. Feynmann, R.P.: Phys. Rev. 56, 340 (1939)

3. Silverman, J.N., van Leuven, J.C.: Chem. Phys. Lett. 7, 640 (1970)

4. Deb, B.M.: Chem. Phys. Lett. 7, 78 (1972)

5. Levine, I.N. Quantum Chemistry, 5th edn. Prentice-Hall, Inc, Upper Saddle River (2000)

6. Atkins, P.W., Friedman, R.S. Molecular Quantum Mechanics, 3rd edn. Oxford University Press, New York (1997)

7. Fan, H., Chen, B.: Phys. Lett. A 203, 95 (1995)

8. Popov, D.: Int. J. Quant. Chem. 69, 159 (1998)

9. Fan, H., Xu, X.: J. Math. Phys. 50, 063303 (2009)

10. Meng, X., Fan, H., Wang, J.: Chin. Phys. B 19(7), 070303/1-4 (2010)

11. Meng, X., Wang, J., Liang, B.: Chin. Phys. B 19(4), 044202/1-5 (2010)

Publisher's Note Springer Nature remains neutral with regard to jurisdictional claims in published maps and institutional affiliations. 